\title{
Towards Understanding of Charging Effects of Conductive Thin-Film Based Phase Plates
}

\author{
R. Janzen ${ }^{1}$, J. Schundelmeier ${ }^{1}$, S. Hettler ${ }^{1}$, M. Dries ${ }^{1}$ and D. Gerthsen ${ }^{1}$
}

\section{Laboratory for Electron Microscopy, Karlsruhe Institute of Technology, Karlsruhe, Germany}

In the past few years, physical phase plates (PP) have become a viable tool to enhance the contrast of weakphase objects in transmission electron microscopy (TEM). Among the most promising phase-shifting devices are thin-film based PPs. Classical approaches, such as the Zernike and Hilbert PPs, are based on the mean inner potential of microstructured thin films [1]. Typically, a thin amorphous carbon (aC)-film is applied with a thickness that is carefully adjusted to induce a well-defined phase shift between the unscattered and the scattered electrons. Unfortunately the illumination causes charging as well as reversible and irreversible changes of the PP material resulting in deviations of the phase shift from its desired distribution. Whereas a novel group of hole-free PPs manages to take advantage of charging $[2,3]$ by a preconditioning procedure before application, conventional thin-film based PPs suffer from those effects.

Our experimental approach to overcome charging of thin-film based PPs was using a material with a high electrical conductivity. However, our Hilbert PPs fabricated from thin films of the metallic glass alloy Pd77.5Cu6.0Si16.5 (PCS) with a high specific conductivity of $1.18 \cdot 10^{6} \mathrm{~S} / \mathrm{m}$ [4] nevertheless show pronounced distortions of the Thon-ring system during illumination with $200 \mathrm{keV}$ electrons. These observations initiated the development of a theoretical model to obtain an improved understanding of charging, which is presented in this work.

Charging is described by assuming a charge-dipole layer to be present at the PP. A possible source for such a dipole layer could be contamination of the PCS film in the illuminated PP region. The electrically insulating contamination layer could capture low-energy secondary electrons generated in the PCS film by the primary electrons. The grounding of the electrically conducting PCS film could eventually lead to the formation of a dipole layer at the interface between the PCS film and the electrically insulating contamination layer. We assume the dipole strength to be proportional to the current density distribution in the back focal plane which can be qualitatively obtained from a diffraction pattern. The proportionality factor for the dipole strength is a fit parameter denoted as phase mask amplitude in the following. The model allows to calculate phase shifts resulting from the assumed dipole layer as a function of position in the back focal plane using the dipole approximation and the high-energy approximation.

As a test of our model we compare power spectra obtained from an experimental image of an aC thinfilm test object with a simulation based on our model. Experimental images were obtained by using a PCS film-based Hilbert PP installed in the back focal plane of a Philips CM 200 FEG/ST electron microscope. The power spectrum of the image is used as experimental reference to be compared with simulated images. We assume the Hilbert PP to be illuminated by the current density distribution given by the diffraction pattern and calculate the phase shift caused by the corresponding dipole layer in $1024 \times 1024$ grid points in the back focal plane. The resulting phase mask is multiplied by different values of the fit parameter (phase mask amplitude) and added to the phase mask of the Hilbert PP within an image simulation procedure which yields a series of simulated power spectra.

Figure 1 shows a montage of a simulated and experimental power spectrum. Two regions, below and above the cut-on frequency, can be distinguished in the power spectra. The cut-on frequency is given by 
the distance between the PP edge and the zero-order beam and is marked by vertical white lines. The complementary behavior of the Thon-rings below and above the cut-on frequency demonstrates the desired phase-shifting properties of the Hilbert PP. It also shows good agreement between experimental and simulated spectra, especially below the cut-on frequency.

The simulated and experimental power spectra are compared pixel by pixel. The sum of all pixel comparisons belonging to one pair of power spectra serves as measure of agreement, which is plotted for different phase mask amplitudes in Figure 2 for the region below the cut-on frequency. Best agreement of almost $75 \%$ is obtained. This is remarkable taking into account that experimental and simulated spectra (based on noisy input for the illumination data) contain noise. We also note that a different value for the phase mask amplitude gives best agreement for the region above the cut-on frequency. The agreement in the outer region can be improved by modifying the background subtraction of the illumination data (diffraction pattern) that has a big influence on the simulation results.

Overall our method seems to be a promising approach to explain phase shift distortions due to charging in thin-film-based PP applications.

[1] R. Danev and K. Nagayama, J. Phys. Soc. Jpn. 73 (2004), p. 2718.

[2] M. Malac et al., Ultramicroscopy 118 (2012), p. 77.

[3] R. Danev et al., PNAS 111 (2014), p. 15635.

[4] B. Chelluri and R. Kirchheim, J. Non-cryst. Solids 54 (1983), p. 107.

[5] Financial support by the Deutsche Forschungsgemeinschaft (DFG).

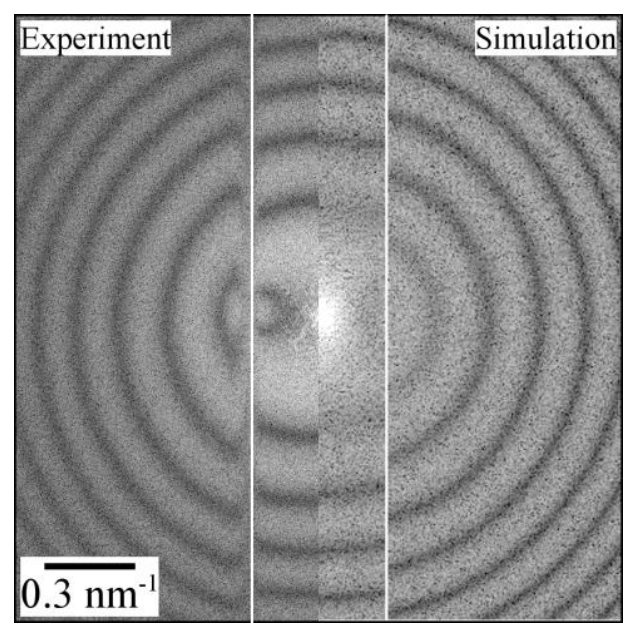

Figure 1. Montage of an experimental power spectrum of an aC test object and a simulated power spectrum. The white lines mark the cut-on frequency of the Hilbert PP which is determined by the distance between the zero-order beam and the edge of the PP.

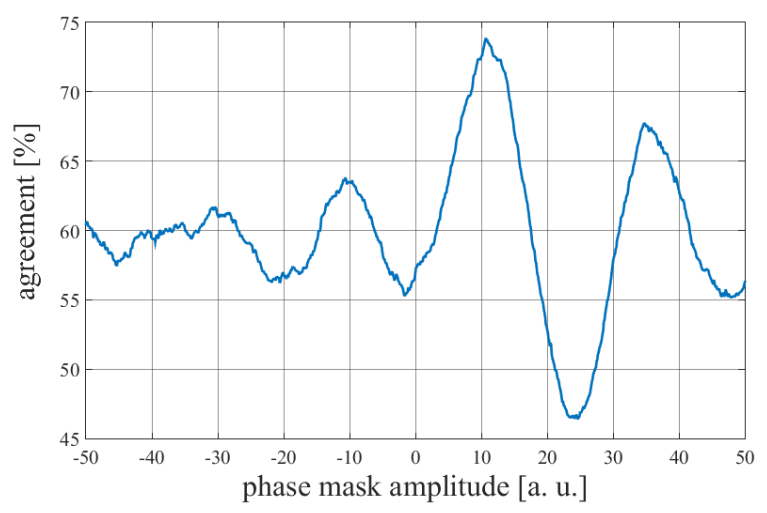

Figure 2. Agreement between experimental and simulated power spectra for spatial frequencies below the cut-on frequency as a function of the fit parameter (phase mask amplitude). 